\title{
Sudden Cardiac Death in Athletes
}

\author{
Andrew D'Silva and Michael Papadakis \\ St George's University of London, St George's University Hospital Foundation NHS Trust, London, UK
}

\begin{abstract}
Physical activity confers substantial health benefits to healthy individuals and patients alike. Occasionally, however, exercise may act as a trigger for arrhythmic death in athletes who harbor an occult pathological substrate. The majority of sudden cardiac deaths (SCDS) in young athletes ( $\leq 35$ years old) are secondary to inherited cardiac diseases, while ischaemic heart disease predominates in older athletes. In the absence of compulsory national or international registries of SCD in athletes, it is difficult to define the exact scale of the problem. In addition, the lack of post-mortem evaluation by pathologists with expertise in cardiac adaptation to exercise and inherited cardiac diseases casts doubt to the reliability of the reported causes. The proposed preventative strategies focus primarily on preventing deaths by cardiovascular evaluation of athletes and the use of automated external defibrillators in athletic venues. Cardiovascular screening of first-degree relatives, though often neglected, has the potential to avert further tragedies given the inherited nature of most conditions predisposing to SCD in the young. This article provides an overview of the epidemiology and causes of SCD in athletes and explores potential prevention strategies.
\end{abstract}

\section{Keywords}

Sudden cardiac death, athlete, pre-participation screening, cardiomyopathy, ion channelopathy, coronary artery disease, risk assessment

Disclosure: Andrew D'Silva and Michael Papadakis have received research grants and work in close collaboration with the charitable organisation Cardiac Risk in the Young (CRY), which supports cardiac screening of all young individuals.

Received: 13 June 2015 Accepted: 9 July 2015 Citation: European Cardiology Review, 2015;10(1):48-53

Correspondence: Michael Papadakis, Department of Cardiovascular and cell sciences, St George's University of London, Cranmer Terrace, London, SW17 ORE.

E: michael.papadakis@sgul.ac.uk

The evidence supporting the beneficial effects of physical activity on health is compelling. Regular exercise reduces cardiovascular mortality by $35 \%$ and all-cause mortality by $33 \%^{1}$ and confers an average of 7 years greater longevity. ${ }^{2}$ Most professional athletes, however, undertake doses of exercise that far exceed those recommended by the current evidence, which has been adopted by national and international health authorities. This is of relevance, as recently published data suggest that, similar to most pharmacological interventions, exercise has an optimal dose above which there is little additional benefit or there may even be harm. ${ }^{3,4}$

Occasionally athletes die suddenly and these highly publicised, tragic instances generate considerable attention from the community at large, given the widely held perception that these individuals are the epitome of fitness and health. In the majority of cases, exercise acts as the trigger of the fatal arrhythmic event rather than being the primary cause. The majority of sudden cardiac deaths (SCDS) in athletes are secondary to quiescent cardiac disease that can potentially be detected during life, galvanising discussions relating to primary and secondary prevention of similar catastrophes. Primary prevention involves the identification of those at risk of sudden cardiac arrest (SCA), through population screening or targeted screening of high-risk individuals with symptoms or family history suggestive of cardiac disease. Implementation of lifestyle interventions and appropriate clinical care can prevent SCA. Secondary prevention relates to improving the probability of survival when an SCA occurs, through the implementation of an effective emergency response plan, with high-quality cardiopulmonary resuscitation (CPR) and prompt use of automated external cardiac defibrillators (AEDS) at its core.

\section{Incidence of Sudden Cardiac Death in Athletes}

The scale of the problem of SCD in athletes is difficult to define, as calculating the precise number of cases (numerator) and defining the exact reference population (denominator) is challenging in the absence of compulsory national or international registries. Studies have arrived at vastly different estimates varying from 1 per 300,000 per year ${ }^{5}$ to 1 per 23,000 per year. ${ }^{6}$ Differences in methodology and selection biases are largely responsible for this variation with available sources for case identification ranging from registries, sporting organisations, parent organisations, media reports or a combination of these. In addition, the estimate of 1 per 23,000 per year included both cases of SCD and survivors of sudden cardiac arrest (SCA), ${ }^{6}$ which is a more reasonable strategy as both SCD and SCA should be targeted by potential preventative strategies.

One large prospective study by Corrado et al. systematically assessed the incidence of SCD in young athletes. ${ }^{7}$ The study is unique as it prospectively studied a well-defined population of athletes and non-athletes in the Veneto region of Italy for 25 years. In contrast to all other studies, owing to the highly organised referral network, all deaths in young (aged 12-35 years) individuals considered to be of cardiac cause were referred for post-mortem evaluation to a single centre and underwent detailed cardiac histopathological evaluation by a small number of expert cardiac pathologists. Moreover, the unique pre-participation screening programme (PPS), which is enforced by law in Italy, ensures that athletes participating in formal competition are subjected to annual medical reviews. Based on the results of this study the incidence of SCD in young athletes was estimated at 1 per 
24,000 per year prior to the initiation of the PPS, ${ }^{8}$ which is similar to that estimated by Drezner et al. in high school student athletes in the US. ${ }^{6}$

A consistent finding across all studies is that male athletes carry a higher risk of SCD than female athletes. The reasons for this are poorly understood. Although higher male participation rates in the most popular sports may partly account for this, gender-specific influences in disease expression are likely to contribute, as similar trends are observed in the general population. ${ }^{9}$ Black athletes also appear to suffer a disproportionately higher incidence of SCD. The National Collegiate Athletic Association in the US reported a three-fold higher risk in African-American athletes compared with Caucasian athletes (1 per 17,000 versus 1 per 58,000), with the highest incidence of SCD in male African-American athletes at 1 per 13,000 per year. ${ }^{10}$

By comparison, it is more difficult to investigate SCD rates in recreational and veteran athletes, as the reporting of these deaths is less consistent and in many cases unlikely to be witnessed. As a result, there are relatively fewer studies evaluating the incidence of SCD in older athletes. A comprehensive, prospective 5-year study of sports-related SCD in the French general population revealed that the overwhelming majority of sports-related SCD occurred among those aged $\geq 35$ years and was greatest in the fifth decade of life. In this study, the overall burden of sports-related SCD was 4.6 deaths per million population per year, with only $6 \%$ occurring in young competitive athletes. ${ }^{11}$ In a more recent study by the same group the incidence of sports-related SCA increased to 21.7 per million population per year when focusing on middle-aged individuals (aged 35-65 years). ${ }^{12}$

\section{Aetiology of SCD in Athletes}

\section{Causes of Sudden Cardiac Death in Young Athletes}

The causes of SCD in athletes are divided based on the age of the athlete; the age of 35 years is used as the cut-off point. In athletes $\leq 35$ years of age, inherited or potentially inherited cardiac diseases account for most SCDS. There is, however, disparity across different registries as to the most common cause of SCD. ${ }^{1}$ In the US registry from the Minneapolis Heart Institute Foundation, hypertrophic cardiomyopathy ( $\mathrm{HCM}$ ) was the most common cause of death, accounting for $36 \%$ of SCDS in this population. Coronary artery anomalies of abnormal origin were next in frequency at $17 \%$ and arrhythmogenic right ventricular cardiomyopathy (ARVC) was responsible for only $4 \%$ of deaths. Other causes of SCD such as myocarditis, ion channelopathies, coronary artery disease (CAD), dilated cardiomyopathy, mitral valve prolapse, aortic stenosis and aortic rupture each accounted for $\leq 6 \%$ of SCDs (see Figure 1). ${ }^{14}$

In comparison, the results of the registry in the Veneto region of Italy place ARVC as the most common cause of SCD (23\%), followed by atherosclerotic CAD (19\%).? Anomalous origin of the coronary arteries accounted for $13 \%$ of SCDS, with mitral valve prolapse contributing $12 \%$, myocarditis $10 \%$ and conduction system disease $8 \%$ (see Figure 1).? Although the Italian registry is smaller in scale compared with the US registry, the accuracy of diagnoses by specialist cardiac pathologists give additional credibility to the results. Variations between the findings in each registry may be explained in part by the differences in the population, differences in methodology relating to data collection and interpretation of autopsy findings and the fact that systematic PPS of athletes in Italy may have prevented SCDs due to HCM through the disqualification of affected athletes.

Figure 1: Comparison of Causes of Sudden Cardiac Death

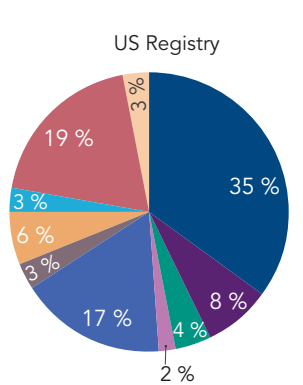

US Military

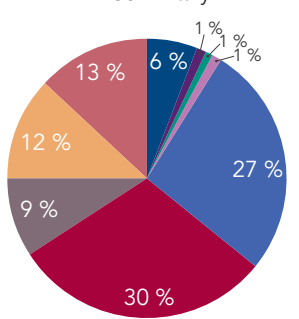

$A R V C=$ arrhythmogenic cardiomyopathy $: C M=$ cardiomyopathy $\cdot \mathrm{HCM}=$ hypertrophic cardiomyopathy; IHD = ischaemic heart disease; $L V H$ = left ventricular hypertrophy; NCAA = National Collegiate Athletic Association; SADS = sudden arrhythmic death syndrome; $S C T=$ sickle cell trait. Reproduced with permission from Harmon et al..$^{13}$ with data taken from Corrado et al. ${ }^{7}$

Figure 2: Yield of Genetic Cardiovascular Conditions from Familial Evaluation Following Sudden Arrhythmic Death Syndrome

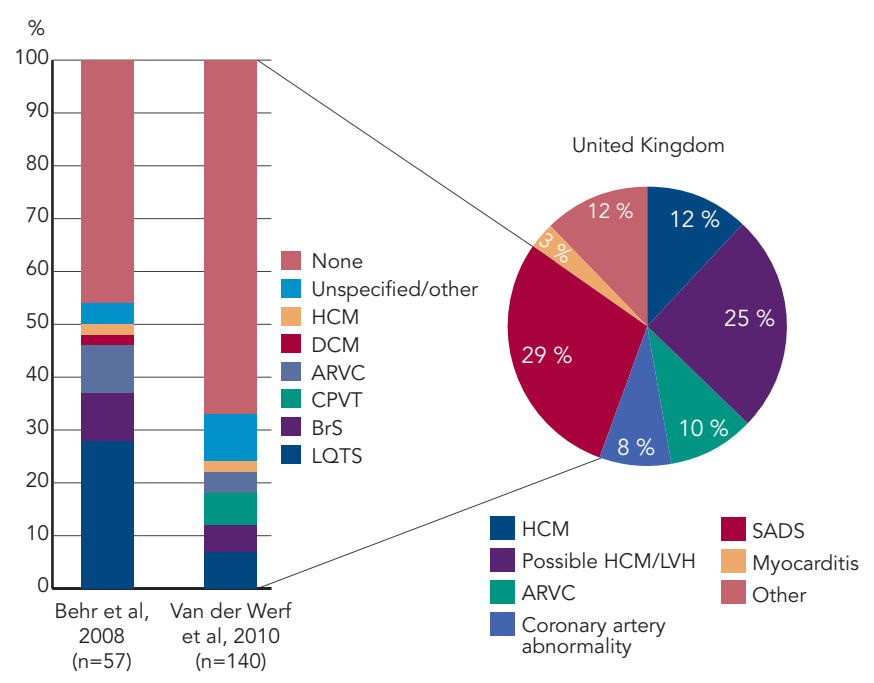

Right: Pie chart showing the causes of sudden cardiac death in 89 sports deaths in young athletes referred to a tertiary cardiac centre in the UK over a duration of12 years. Left: Bar chart showing the yield of genetic cardiovascular conditions from familial evaluation series following sudden unexplained death and/or SADS. ARVC = arrhythmogenic right ventricular cardiomyopathy; BrS = Brugada syndrome; $C P V T=$ catecholaminergic polymorphic ventricular tachycardia; DCM = dilated cardiomyopathy; $H C M=$ hypertrophic cardiomyopathy; LQTS = Iong QT syndrome; LVH = left ventricular hypertrophy; $M I=$ myocardial infarction; SADS = sudden arrhythmic death syndrome. Data taken from de Noronha et al., ${ }^{15}$ Behr et al. ${ }^{17}$ and Van der Werf et al. ${ }^{45}$

\section{Contemporary Studies of Causes of SuddenCardiac Death in Athletes}

Contemporary studies have further added to the diversity of SCD causes in athletes and highlighted the complexity of the subject. Post-mortem examination of 89 young athletes in the UK suffering SCD revealed a normal heart as the most common finding, implicated in almost a third (29\%) of deaths. ${ }^{15}$ Other novel entities included idiopathic left ventricular hypertrophy (LVH) and idiopathic fibrosis accounting for $25 \%$ and $7 \%$ of SCDS, respectively. Established cardiomyopathies such as HCM and 
ARVC accounted for $22 \%$ of cases, while coronary artery pathology was present in $8 \%$ of deaths (see Figure 2). ${ }^{15}$ Although the high prevalence of autopsy-negative SCDS in the UK study may be in part attributed to significant referral bias, further studies have reported similar results. Of the 45 SCDs in US college athletes, a structurally normal heart was the most common finding at post-mortem accounting for $31 \%$ of cases, while idiopathic LVH was present in $8 \% .{ }^{13}$ Similarly, a 25 -year review of deaths in young military recruits in the US, identified autopsy-negative sudden unexplained death in $30 \%$ of cases (see Figure 1). ${ }^{16}$

Recognition of a normal heart on autopsy, also referred as sudden arrhythmic death syndrome (SADS), is of paramount importance given its association with inherited ion channelopathies. ${ }^{17}$ Studies on familial evaluation after a SADS death have demonstrated evidence of an ion channelopathy or cardiomyopathy in up to $50 \%$ of families (see Figure 2). ${ }^{17}$ In addition, the significance of idiopathic LVH and idiopathic fibrosis remains uncertain and postulated theories include being innocent bystanders, pathological variants of physiological LVH in genetically predisposed individuals or part of the $\mathrm{HCM}$ spectrum. ${ }^{18}$ In athletes with normal hearts or autopsy findings of uncertain significance the contribution of severe metabolic or electrolyte disturbances and heatstroke must also be taken into consideration as potential causes of sudden death. One study examining life-threatening and fatal events occurring during endurance races in Israel over a 6-year period found that heatstroke was responsible for the majority of events. ${ }^{19}$

\section{Causes of Sudden Cardiac Death in Older Athletes}

The predominant cause of SCD in athletes aged $>35$ years is atherosclerotic coronary artery disease (CAD), identified in more than $80 \%$ of cases. $^{20}$ Acute exercise can lead to transient activation of the coagulation system, which promotes a pro-thrombotic environment. ${ }^{21}$ Additional metabolic and haemodynamic changes during exercise may contribute to stress-related plaque rupture observed in sportsrelated victims of $S C D .22$ On post-mortem examination, coronary atheroma with significant luminal obstruction (>75\%) may be present, ${ }^{23,24}$ with associated features indicative of acute or chronic myocardial infarction. However, plaque rupture, thrombosis, acute infarction and fibrosis are not prerequisites for the cause of death to be attributable to CAD. In such cases, the presumed mode of SCD is sudden ventricular arrhythmia due to myocardial perfusion-demand mismatch and resultant ischaemia. ${ }^{25}$

\section{Prevention and Management of SCD in Athletes}

The steady trickle of SCD in athletes galvanises discussions relating to potential prevention measures in order to avert such tragedies. Proposed strategies include targeted evaluation of high-risk individuals, pre-participation cardiac evaluation of all athletes to identify quiescent conditions predisposing to exercise-related SCD, comprehensive evaluation of both the victim of SCD or SCA and most importantly of the family relatives who may harbor the same condition, and emergency response planning to ensure prompt resuscitation of cardiac arrests.

\section{Primary Prevention Strategies}

\section{Targeted Evaluation of High-risk Individuals}

Athletes with symptoms suggestive of cardiac disease or a family history of potentially inherited conditions or SCD should receive comprehensive evaluation. Of particular concern are new-onset exertional symptoms such as chest pain, shortness of breath, presyncope and syncope. This strategy in isolation is of limited value as the majority of victims of SCD are asymptomatic and SCD/SCA is commonly the first presentation. 15,26

\section{Pre-participation Cardiac Evaluation of Young Athletes}

Pre-participation cardiac screening using the 12-lead ECG remains a controversial issue. Data from the Italian national screening programme in young athletes reported a $90 \%$ reduction in SCD. ${ }^{8}$ In contrast, Israel's introduction of mandatory PPS did not appear to alter the incidence of SCD in athletes. ${ }^{27}$ However, a possible explanation for the discrepancy is the difference in data collection methods between the two studies. The Israeli study collected mortality data from Israel's two main newspapers and estimated the number of athletes in the population (denominator) for the earlier years of the study period. ${ }^{27}$ By comparison, the Italian study had well-defined population data and a robust national reporting system of SCD cases. ${ }^{8}$ Additional concerns centre on the inability of screening to identify a considerable proportion of conditions predisposing athletes to SCD, the false-positive ECGS, feasibility and cost-effectiveness.

The Italian PPS programme is mandatory for all young competitive athletes aged 12-35 years. The first tier of evaluation involves history (including family history), physical examination and 12-lead ECG. If no abnormalities are detected, the athlete is eligible for competition. Those with positive findings suggestive of possible cardiac disease are investigated further based on local protocols and the condition suspected. If this second tier of targeted evaluation reveals no evidence of cardiovascular disease, the athlete is eligible for competition. Where a cardiovascular disease is diagnosed, the athlete is managed according to established protocols (see Figure $3 A$ ). ${ }^{28}$

The ECG is likely to raise suspicion of quiescent cardiac disease in the majority of individuals with cardiomyopathies and is the primary diagnostic tool for ion-channelopathies and ventricular pre-excitation. However, up to $10 \%$ of patients with $\mathrm{HCM}^{29} 20 \%$ with $\mathrm{ARVC}^{30}$ and $25 \%$ with long-QT syndrome ${ }^{31}$ may exhibit a normal ECG. In addition, the resting ECG will not detect the vast majority of individuals with coronary artery anomalies.

The false-positive ECG rate using the European Society of Cardiology (ESC) criteria has been reported to be as high as $40 \%$ in certain athletic cohorts, which raises concerns relating to the burden of unnecessary investigations or erroneous disqualification. Recent studies in large cohorts of young athletic individuals have resulted in refinement of the ECG criteria considered to denote an abnormal result, with a considerable improvement of the ECG specificity. ${ }^{32,33}$ Further research is necessary, particularly in athletes of non-Caucasian ethnicity, as the false positive ECG rate in athletes of African-Caribbean descent remains over $15 \%$ and limited data exist on other ethnicities. ${ }^{34}$

Widespread adoption of PPS is also hindered by the lack of expertise and facilities. With the exception of Italy, where a mandatory, National screening programme has been in place since 1980, in most other countries screening of athletes is fragmented and dependent on the availability of local expertise and individual sporting organisation mandates. Finally, studies on the cost-effectiveness of PPS are fairly limited and most rely on theoretical projection models and arbitrary assumptions relating to the life years saved by identifying a potentially life-threatening condition. In most countries where the health service is already burdened by limited finances and resources, the implementation of a state-funded, national screening programme to identify silent cardiac diseases in young athletes seems unattainable. 
Figure 3: Pre-participation Screening Protocols for Young Competitive Athletes (A) and Older Adults (B) Undertaking High-intensity Physical Activity

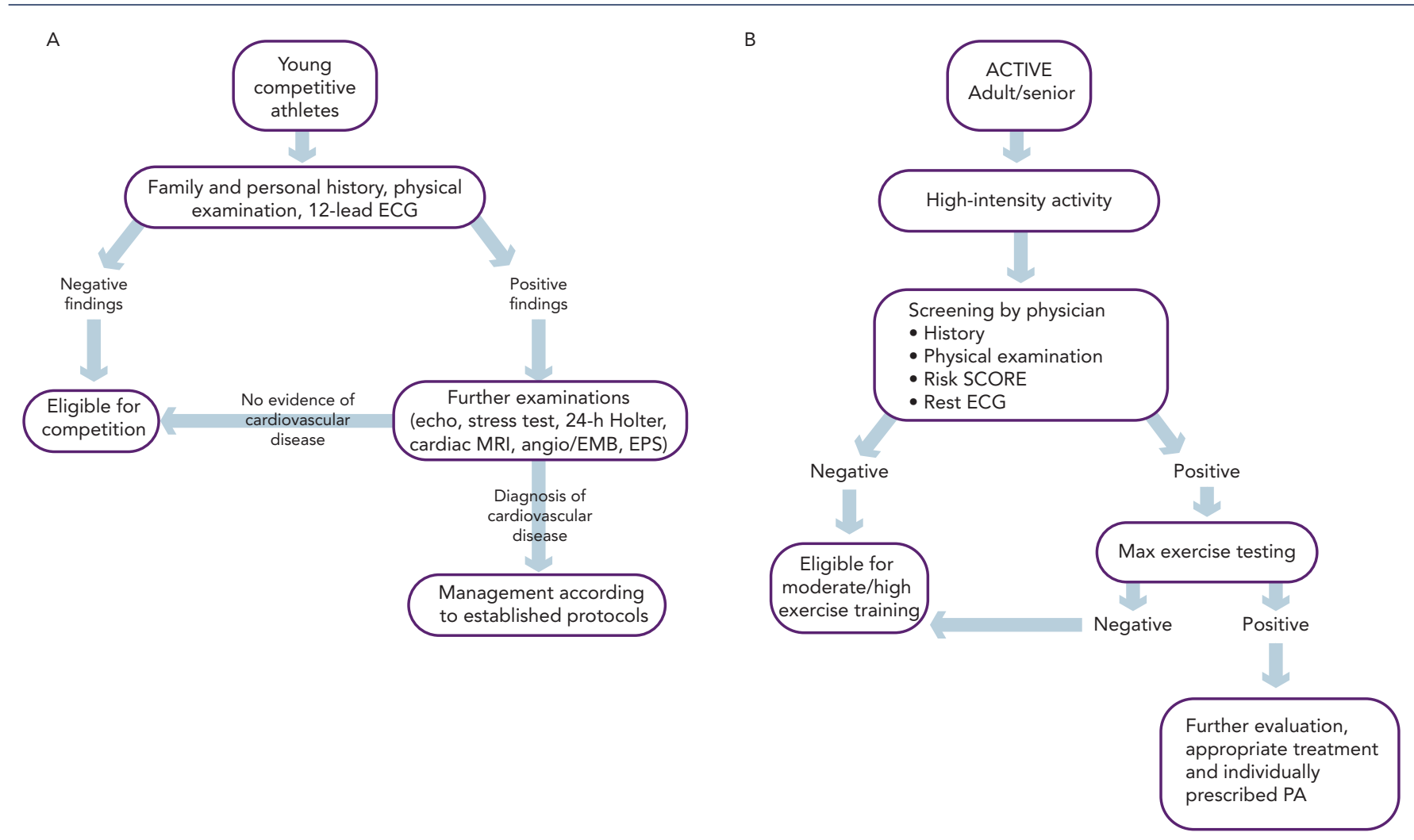

$E M B=$ endomyocardial biopsy; EPS = electrophysiological study; $P A=$ physical activity; SCORE = Systematic Coronary Risk Evaluation. Reproduced with permission from Corrado et al. ${ }^{4}$ and adapted from Borjessen et al. ${ }^{37}$

There are, however, convincing arguments for alternative funding sources, with charitable organisations subsidising the screening of young athletes who wish to be screened for self-protection. ${ }^{35}$

\section{Pre-participation Cardiac Evaluation of Older Athletes}

The rapidly expanding population of older veteran or amateur athletes who compete in high-intensity events underscores the importance of preventing SCD in this age group. The resting 12-lead ECG is of limited value in screening senior athletes, as CAD is the main cause of SCD. ${ }^{36}$ The sports Cardiology section of the European Association of Cardiovascular Prevention and Rehabilitation published recommendations relating to screening practices, which take into account the burden of cardiovascular risk factors, pre-existing fitness levels and intended level of exercise. ${ }^{37}$ The assessment of athletes undertaking high intensity physical activity incorporates the assessment of cardiovascular risk factors, symptoms and family history; physical examination; resting 12-lead ECG; and the Systematic Coronary Risk Evaluation (SCORE) to assess the 10-year risk of a fatal cardiovascular event. Any abnormalities arising from this initial assessment, including a SCORE 10-year risk of $\geq 5 \%$, requires maximal exercise ECG testing. However, it is well recognised that the sensitivity of exercise ECG stress testing in a screening setting with predominantly asymptomatic individuals is poor $(25 \%)^{38}$ and the false-positive rates are high, particularly in women. ${ }^{39}$ Nevertheless, a negative maximal exercise ECG stress test provides eligibility for moderate/high exercise training and a positive test result will require further investigation for underlying CAD, the gold standard test being invasive coronary angiography (see Figure 3B).

An area deserving further consideration is whether computed tomography coronary artery calcium scoring (CACS) can provide a
Figure 4: Differences Between the Autopsy Conclusions of Referring General Pathologists and an Expert Cardiac Pathologist

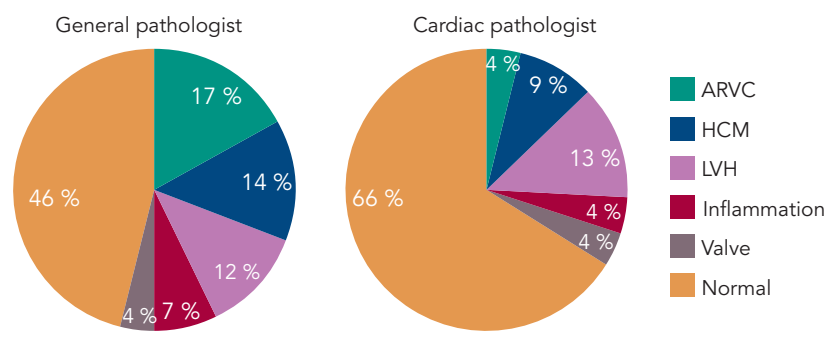

$A R V C=$ arrhythmogenic cardiomyopathy; $H C M=$ hypertrophic cardiomyopathy; $L V H=l$ left ventricular hypertrophy. Data taken from de Noronha et al. ${ }^{41}$

better risk prediction of serious cardiovascular events, as high calcium scores appear to predict increased risk for cardiovascular events in the general population, ${ }^{24}$ including asymptomatic individuals. ${ }^{40}$ Obstacles to the wider implementation of CACS as a screening tool include radiation exposure (though minimal at 1.5-3.0 mSv), availability and cost.

\section{Evaluation of the Patient and the Family}

The inherited nature of most conditions predisposing to SCD in young athletes highlights the importance of comprehensive post-mortem evaluation of the index case as well as cardiovascular screening of first-degree relatives.

The importance of a histopathological examination by a cardiac pathologist with expertise in the morphological adaptations of the athletic heart and disease phenotypes implicated in SCD in athletes cannot be overstated, as false conclusions may misguide familial evaluation or offer false reassurance to surviving relatives and 
Figure 5: Principles of Managing SCD

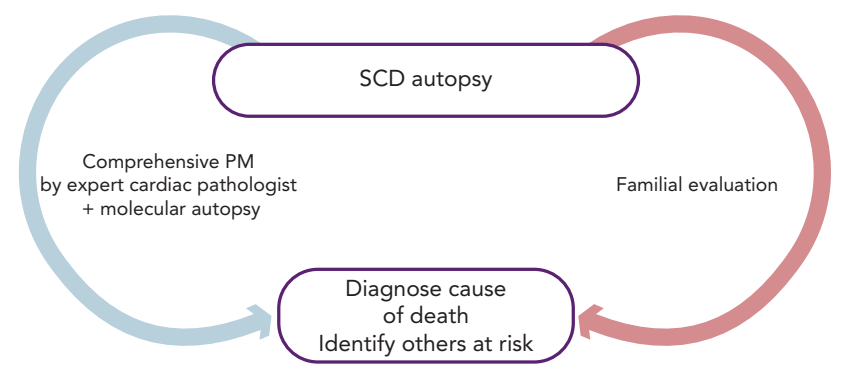

$P M=$ post mortem; $S C D=$ sudden cardiac death

dissuade physicians from initiating familial screening. When comparing the cause of death attributed to a series of SCDS, there was a disparity in $41 \%$ of cases between the referring pathologists and the specialist pathologist ${ }^{41}$. Referring pathologists were more inclined to diagnose cardiomyopathy than morphologically normal heart, with only $63 \%$ of normal hearts being described correctly (see Figure 4). Examples of misdiagnoses can include post-mortem coronary artery collapse with mild atheroma being mislabelled as CAD; overdiagnosis of ARVC where right ventricular fatty infiltration is related to obesity, alcohol misuse or other causes; and overdiagnosis of HCM in hypertrophied hearts despite the absence of myocyte diasarray. ${ }^{18} \mathrm{~A}$ guideline has been developed by the Association for European Cardiovascular Pathology to reduce variation in practice and to provide a minimum standard, ensuring accurate, systematic examination and sampling in the autopsy investigation of SCD. ${ }^{42}$

The retention at post mortem of blood or splenic tissue allows for genetic testing, which is referred to as molecular autopsy. Genetic mutations can be identified and may be highly relevant and potentially informative as to the possible cause of SCD, and can facilitate clinical and genetic screening of family members to identify those at risk. Molecular autopsy opens the possibility to investigate ion-channel disorders such as long-QT syndrome, catecholaminergic polymorphic ventricular tachycardia and Brugada syndrome at post mortem, where the heart is unlikely to demonstrate any structural abnormality, also referred to as SADS.

Complementary to the process of establishing an accurate cause of death is the process of investigating first-degree family members (see Figure 5). Where a clear cause of death is established by histopathological examination or molecular autopsy, targeted investigations suited to the detection of that condition can be applied to the relatives of the SCD patient. In cases where no clear cause has been found at autopsy, or the autopsy revealed a morphologically normal heart, investigations are used sequentially to assess for the presence of cardiomyopathy or an inheritable arrhythmogenic condition. First-line assessment includes history, physical examination, resting 12-lead ECG, transthoracic echocardiography, exercise tolerance testing and 24h-Holter ECG monitoring. The use of further tests is predicated upon the findings of the first-line assessment. Up to $25 \%$ of relatives of patients with SADS are diagnosed with a previously unsuspected inherited cardiac condition ${ }^{17}$ and interventions ranging from simple lifestyle modification advice to the implantation of a prophylactic implantable cardioverterdefibrillator can reduce the risk of further fatalities.

\section{Secondary Prevention Strategies Emergency Response Planning}

A comprehensive medical action plan that is rehearsed on a regular basis is essential to ensure the best possible outcome in the context of SCA in sports arenas. ${ }^{43}$ The importance of an effective emergency response plan is underscored by studies that have demonstrated that prompt CPR and defibrillation of athletes (within 5 minutes) resulted in survival rates in excess of $60 \% .^{6}$ The same message is reiterated by studies of sports-related SCA in the general population, where a witnessed event, prompt CPR and the use of a cardiac defibrillator ventricular fibrillation were associated with higher survival to hospital discharge. ${ }^{11,12}$

The Fédération Internationale de Football Association (FIFA) support and promote a standardised and consistent level of advanced life support and emergency medical care on the football field, including a FIFA universal medical emergency bag and an '11-step plan to prevent SCD'. The 'FIFA 11 steps' include primary prevention with PPS, training of personnel on CPR, availability of AEDS, rehearsal of the emergency medical plan on an annual basis, equipment checks, logistical planning and protocols designed to enhance the resuscitation team's performance. ${ }^{44}$

\section{Conclusion}

Exercise confers substantial health benefits to the vast majority of individuals but may serve as a trigger for arrhythmic death in a small minority who harbor an occult pathological substrate. Currently, PPS of young competitive athletes is recommended and supported by many societies, associations and sporting bodies around the world. Groups, arguably at greater risk of SCD, not receiving as much attention and having less access to screening include school children playing sport, senior athletes and those undertaking grassroots, amateur and recreational sports. The aspiration is that future research in this area will improve prediction tools for SCD risk in athletes and institute tailored management, including individualized exercise prescription, to prevent fatalities. However, there will always be a need to ensure that, in the event of SCA, an appropriate emergency response plan is in place and a defibrillator is readily accessible. Finally, the SCA or SCD of a young individual should instigate comprehensive evaluation of the patient and the family to ensure that relatives at potential risk are identified and treated appropriately.
1. Nocon M, Hiemann $\mathrm{T}$, Muller-Riemenschneider $\mathrm{F}$, et al. Association of physical activity with all-cause and cardiovascular mortality: a systematic review and metaanalysis. Eur J Cardiovasc Prev Rehabil 2008:15(3):239-46.

2. Chakravarty EF, Hubert HB, Lingala VB, Fries JF. Reduced disability and mortality among aging runners: a 21-year longitudinal study. Arch Intern Med 2008;168(15):1638-46.

3. Armstrong ME, Green J, Reeves GK, et al. Frequent physica activity may not reduce vascular disease risk as much as moderate activity: large prospective study of women in the United kingdom. Circulation 2015:131(8):721-9.

4. Schnohr P, O'Keefe JH, Marott JL, et al. Dose of jogging and Iong-term mortality: the Copenhagen City Heart Study. J Am coll Cardiol 2015:65(5):411-9.

5. Van Camp SP, Bloor CM, Mueller FO, et al. Nontraumatic sports death in high school and college athletes. Med Sci Sports Exerc 1995:27(5):641-7.

6. Drezner JA, Rao AL, Heistand t, et al. Effectiveness of emergency response planning for sudden cardiac arrest in United States high schools with automated external defibrillators. Circulation 2009;120(6):518-25.

7. Corrado D, Basso C, Rizzoli G, et al. Does sports activity enhance the risk of sudden death in adolescents and youn adults? J Am Coll Cardiol 2003;42(11):1959-63

8. Corrado D, Basso C, Pavei A, et al. Trends in sudden cardiovascular death in young competitive athletes after implementation of a preparticipation screening program. JAMA 2006;296(13):1593-601.

9. Papadakis M, Sharma S, Cox S, et al. The magnitude of sudden cardiac death in the young: a death certificate-based review in England and Wales. Europace 2009;11(10):1353-8.

10. Harmon $\mathrm{KG}$, Asif IM, Klossner D, Drezner JA. Incidence of sudden cardiac death in national collegiate athletic association athletes. Circulation 2011;123(15):1594-600.

11. Marijon E, Tafflet M, Celermajer DS, et al. Sports-related sudden death in the general population. Circulation 2011; 124(6):672-81

12. Marijon E, Uy-Evanado A, Reinier K, et al. Sudden cardiac arrest during sports activity in middle age. Circulation 2015;131(16):1384-91.

13. Harmon KG, Drezner JA, Maleszewski JJ, et al. Pathogeneses of sudden cardiac death in national collegiate athletic association athletes. Circ Arrhythm Electrophysiol 2014;7(2):198-204.

14. Maron BJ, Doerer JJ, Haas TS, et al. Sudden deaths in young competitive athletes: analysis of 1866 deaths in the United 
States, 1980-2006. Circulation 2009;119(8):1085-92.

15. de Noronha SV, Sharma S, Papadakis M, et al. Aetiology of sudden cardiac death in athletes in the United Kingdom: a pathological study. Heart 2009:95(17):1409-14.

16. Eckart RE, Scoville SL, Campbell $C L$, et al. Sudden death in young adults: a 25-year review of autopsies in military recruits. Ann Intern Med 2004;141(11):829-34.

17. Behr ER, Dalageorgou C, Christiansen M, et al. Sudden arrhythmic death syndrome: familial evaluation identifies inheritable heart disease in the majority of families. Eur Heart 2008;29(13): 1670-80.

18. Papadakis M, Raju H, Behr ER, et al. Sudden cardiac death with autopsy findings of uncertain significance: potential with autopsy findis of union 2013;6(3):588-96.

19. Yankelson L, Sadeh B, Gershovitz L, et al. Life-threatening events during endurance sports: is heat stroke more prevalent than arrhythmic death? I Am Coll Cardiol 2014;64(5):463-9.

20. Maron BJ, Epstein SE, Roberts WC. Causes of sudden death in competitive athletes. J Am Coll Cardiol 1986:7(1):204-14.

21. Koenig W, Ernst E. Exercise and thrombosis. Coron Artery Dis 2000;11(2):123-7.

22. Chugh SS, Weiss JB. Sudden cardiac death in the older athlete. J Am Coll Cardiol 2015:65(5):493-502.

23. Tabib A, Miras A, Taniere P, Loire R. Undetected cardiac lesions cause unexpected sudden cardiac death during occasional sport activity. A report of 80 cases. Eur Heart 1999;20(12):900-3.

24. Möhlenkamp S, Lehmann N, Breuckmann F, et al. Running: the risk of coronary events: Prevalence and prognostic relevance of coronary atherosclerosis in marathon runners. Eur Heart J 2008;29(15):1903-10.

25. Sheppard MN. Aetiology of sudden cardiac death in sport: a histopathologist's perspective. Br I Sports Med 2012;46 (Suppl 1):i15-21.

26. Maron BJ, Shirani J, Poliac LC, et al. Sudden death in young competitive athletes. Clinical, demographic, and pathological profiles. JAMA 1996;276(3):199-204.

27. Steinvil $A$, Chundadze $T$, Zeltser $D$, et al. Mandatory electrocardiographic screening of athletes to reduce their risk for sudden death proven fact or wishful thinking?
J Am Coll Cardiol 2011;57(11):1291-6.

28. Pelliccia A, Fagard R, Bjornstad $\mathrm{HH}$, et al. Recommendations for competitive sports participation in athletes with cardiovascular disease: a consensus document from the Study Group of Sports Cardiology of the Working Group of Cardiac Rehabilitation and Exercise Physiology and the Working Group of Myocardial and Pericardial Diseases of the European Society of Cardiology. Eur Heart J 2005;26(14):1422-45.

29. Maron BJ. Hypertrophic cardiomyopathy: a systematic review. JAMA 2002;287(10):1308-20.

30. Marcus Fl. Electrocardiographic features of inherited diseases that predispose to the development of cardiac arrhythmias, long QT syndrome, arrhythmogenic right ventricular cardiomyopathy/dysplasia, and Brugada syndrome. J Electrocardiol 2000;33 Suppl:1-10.

31. Goldenberg I, Horr S, Moss AJ, et al. Risk for life-threatening cardiac events in patients with genotype-confirmed long-QT syndrome and normal-range corrected QT intervals. J Am Coll Cardiol 2011;57(1):51-9.

32. Gati S, Sheikh N, Ghani S, et al. Should axis deviation or atrial enlargement be categorised as abnormal in young athletes? The athlete's electrocardiogram: time for re-appraisal of markers of pathology. Eur Heart J 2013;34(47):3641-8.

33. Zaidi $A$, Ghani $S$, Sheikh $N$, et al. Clinical significance of electrocardiographic right ventricular hypertrophy in athletes: comparison with arrhythmogenic right ventricular cardiomyopathy and pulmonary hypertension. Eur Heart J cardiomyopathy and

34. Sheikh N, Papadakis M, Ghani S, et al. Comparison of electrocardiographic criteria for the detection of cardiac abnormalities in elite black and white athletes. Circulation 2014;129(16):1637-49.

35. Papadakis M, Carre F, Kervio G, et al. The prevalence, distribution, and clinical outcomes of electrocardiographic repolarization patterns in male athletes of African/AfroCaribbean origin. Eur Heart J 2011;32(18):2304-13.

36. La Gerche A, Baggish AL, Knuuti J, et al. Cardiac imaging and stress testing asymptomatic athletes to identify those at risk of sudden cardiac death. JACC Cardiovasc Imaging 2013;6(9):993-1007.

37. Borjesson M, Urhausen A, Koulid E, et al. Cardiovascular evaluation of middle-aged/ senior individuals engaged in leisure-time sport activities: position stand from the sections of exercise physiology and sports cardiology of the European on and Rehabilitation. Eur J Cardiovasc Prev Rehabil 2011;

38. Froelicher VF, Fearon WF, Ferguson CM, et al. Lessons learned from studies of the standard exercise ECG test. Chest 1999;116(5):1442-51.

39. Gianrossi R, Detrano R, Mulvihill D, et al. Exercise-induced ST depression in the diagnosis of coronary artery disease. A meta-analysis. Circulation 1989;80(1):87-98.

40. Nasir K, Clouse M. Role of nonenhanced multidetector CT coronary artery calcium testing in asymptomatic an symptomatic individuals. Radiology 2012;264(3):637-49.

41. de Noronha SV, Behr ER, Papadakis M, et al. The importance of specialist cardiac histopathological examination in the investigation of young sudden cardiac deaths. Europace 2014;16(6):899-907.

42. Basso C, Burke M, Fornes P, et al. Guidelines for autopsy investigation of sudden cardiac death. Virchows Arch 2008;452(1):11-8

43. Borjesson M, Dugmore D, Mellwig KP, et al. Time for action regarding cardiovascular emergency care at sports arenas: a lesson from the Arena study. Eur Heart J 2010:31(12): 1438-41.

44. Dvorak J, Kramer EB, Schmied CM, et al. The FIFA medical emergency bag and FIFA 11 steps to prevent sudden cardiac death: setting a global standard and promoting consistent football field emergency care. Br I Sports Med 2013;47(18):1199-202.

45. van der Werf C, Hofman N, Tan HL, et al. Diagnostic yield sudden unexplained death and aborted cardiac arrest in the young: the experience of a tertiary referral center in The Netherlands. Heart Rhythm 2010;7(10):1383-9.

46. Corrado D, Pelliccia A, Bjornstad HH, et al. Cardiovascular pre-participation screening of young competitive athletes for prevention of sudden death: proposal for a common European protocol. Consensus Statement of the Study Group of Sport Cardiology of the Working Group of Cardiac Rehabilitation and Exercise Physiology and the Working Group of Myocardial and Pericardial Diseases of the European Society of Cardiology. Eur Heart $J$ 2005:26(5):516-24. 